\title{
Frequência de intercorrências gestacionais e obstétricas em crianças com transtorno do espectro autista em uma escola especializada em Salvador, Bahia
}

\section{The frequency of gestational and obstetric intercurrences in children with autistic spectrum disorder in a specialized school in Salvador, Bahia}

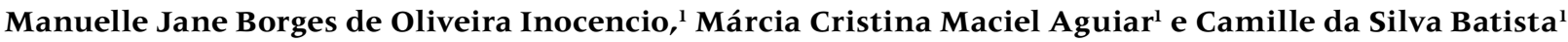 \\ Universidade do Estado da Bahia
}

\section{Palavras-chave:}

Transtorno do espectro autista; Complicações na gravidez; Assistência perinatal.

\section{Resumo}

Introdução: o transtorno do espectro autista é uma condição de neurodesenvolvimento caracterizada por alterações na comunicação, interação social e padrões repetitivos e estereotipados de comportamento, segundo o Manual diagnóstico e estatístico de transtornos mentais $5^{a}$ edição. Suspeita-se que a etiologia desse transtorno seja multifatorial, associada a fatores neurológicos, genéticos e ambientais. Objetivo: identificar intercorrências gestacionais e obstétricas, bem como verificar a sua prevalência, na gestação de crianças com transtorno do espectro autista de uma escola especializada. Métodos: estudo descritivo, com desenho transversal, não experimental e período de referência retrospectivo, realizado a partir da coleta de dados em prontuários médicos de crianças com transtorno do espectro autista matriculadas em uma escola especializada na cidade de Salvador - BA. Resultados: foram colhidos dados de 209 prontuários médicos, porém foram excluídos 65 por estarem preenchidos de forma incompleta. O número amostral total foi de 144 prontuários. Desses, 41,66\% não tiveram nenhuma intercorrência gestacional ou obstétrica e 58,33\% tiveram algum tipo de intercorrência. No grupo com intercorrências ocorreram 35 diferentes alterações, com alguns indivíduos apresentando mais que uma alteração (média de 1,69 alterações por criança). Conclusões: nas gestações de crianças autistas existe grande concentração de intercorrências acontecendo. Prematuridade, sangramento durante a gestação e sofrimento fetal foram as intercorrências mais prevalentes na gestação das crianças com transtorno do espectro autista da escola especializada pesquisada. Crianças com autismo grave tiveram maior percentual de intercorrências gestacionais e obstétricas do que crianças com autismo leve.

\section{Keywords:}

Autism spectrum disorder; Pregnancy complications; Perinatal care.

\begin{abstract}
Introduction: autism spectrum disorder is a condition of neurodevelopment characterized by changes in communication, social interaction and repetitive, stereotyped patterns of behavior, according to the Diagnostic and Statistical Manual of Mental Disorders. It is suspected that the etiology of this disorder is multifactorial, associated with neurological, genetic and environmental factors. Objective: to identify gestational and obstetric intercurrences, as well as to verify their prevalence in the gestation of children with autism spectrum disorder from a specialized school. Methods: a descriptive, cross-sectional and non-experimental study with retrospective reference period, based on data collection in medical records of children with autism spectrum disorder enrolled in a specialized school in the city of Salvador - BA. Results: the data was collected from 209 medical records, but 65 were excluded due to incomplete filling. The total sample was 144 . Of these, $41.66 \%$ had no gestational and obstetric intercurrence and $58.33 \%$ had some kind of intercurrence. In the intercurrence group, 35 different alterations were found, with some individuals presenting more than one (average of 1.69 alterations per child). Conclusions: this study found that pregnancies of children with autism spectrum disorder had a high prevalence of intercurrences. Prematurity, bleeding during gestation and fetal distress were the most prevalent intercurrences in the gestation of children with autism spectrum disorder at the specialized school investigated. Children with severe autism had a higher prevalence of intercurrences when compared to children with mild autism.
\end{abstract}




\section{INTRODUÇÃO}

O transtorno do espectro autista (TEA) é uma condição de neurodesenvolvimento caracterizada por alterações na comunicação, interação social e padrões repetitivos e estereotipados de comportamento, segundo o Manual diagnóstico e estatístico de transtornos mentais (DSM5). ${ }^{1}$ Eles incluem prejuízo na comunicação e interação social, além de padrões limitados e repetitivos de comportamento, interesses ou atividades. ${ }^{2}$ É um transtorno que acomete precocemente mecanismos cerebrais de sociabilidade básica. ${ }^{3}$

O conceito de autismo infantil veio sofrendo diversas modificações ao longo do tempo e a expressão 'transtorno do espectro autista' surgiu devido à consciência de que as manifestações comportamentais são distintas, heterogêneas e que existem vários graus distintos de acometimento, constituindo um espectro. Esse termo foi adotado pelo DSM-5 e colocado sob o título de desordem do neurodesenvolvimento. ${ }^{2} \mathrm{O}$ TEA apresenta prevalência elevada tanto no Brasil como no mundo. ${ }^{3} \mathrm{O}$ fato de sua etiologia ainda permanecer desconhecida dificulta uma cura definitiva. ${ }^{3}$ Embora a neuropatologia permaneça indefinida, estudos têm demonstrado anormalidades funcionais do cérebro que sugerem que o período relevante para o seu desenvolvimento seja o intrauterino e que a patogênese pode se iniciar no período pré-natal. ${ }^{4}$

Demonstrou-se então uma necessidade de pesquisar melhor as bases genéticas, os fatores de risco pré-diagnóstico e as contribuições ambientais, tanto pré como pós-natais nos indivíduos com TEA. ${ }^{5}$

Mediante esses aspectos, o presente estudo teve como objetivo identificar intercorrências gestacionais e obstétricas, bem como verificar a sua prevalência, na gestação de crianças com TEA de uma escola especializada.

\section{MÉTODO}

Trata-se de um estudo descritivo, com desenho transversal, não experimental e período de referência retrospectivo. A população de estudo é composta por crianças com TEA matriculadas em uma escola especializada na cidade de Salvador - BA, a Associação Amigos dos Autistas (AMA-BA). A amostra foi de conveniência, na qual foram avaliados os prontuários dos alunos matriculados, salientando que todos eles possuem diagnóstico confirmado de TEA. Foram excluídos os prontuários que não estavam corretamente preenchidos com as variáveis a serem estudadas.

Os prontuários analisados estão na própria instituição de ensino desde a admissão do aluno como forma de acompanhamento médico.
As variáveis coletadas nos prontuários foram: idade (em faixas etárias); sexo; cor; intercorrências durante a gestação e nascimento; e grau de autismo.

O grau de autismo foi descrito a partir da Childhood Autism Rating Scale (CARS). Trata-se de um dos instrumentos mais utilizados para a avaliação de crianças autistas, sendo apropriada para uso em qualquer criança acima de 2 anos de idade. É uma escala composta por 15 itens que visam identificar crianças com autismo, e sua importância é baseada na capacidade de diferenciar os graus de comprometimento do autismo entre leve-moderado e grave. Os itens são compostos por domínios que geralmente são afetados no autismo, sendo eles:

a) relações pessoais;

b) imitação;

c) resposta emocional;

d) uso corporal;

e) uso de objetos;

f) resposta a mudanças;

g) resposta visual;

h) resposta auditiva;

i) resposta e uso do paladar;

j) medo ou nervosismo;

k) comunicação verbal;

l) comunicação não verbal;

m) nível de atividade;

n) nível e consistência da resposta intelectual;

o) impressões gerais. ${ }^{6}$

Os dados coletados foram analisados descritivamente, avaliando-se a frequência de cada intercorrência gestacional e obstétrica encontrada, bem como sua relação com os graus de TEA nas crianças. Foi utilizado o método estatístico simples, avaliando a distribuição das porcentagens nos grupos avaliados. Para caracterização da população foi realizada análise descritiva das variáveis. O banco de dados e a sua análise foram realizados no programa Excel ${ }^{\circledR}$.

O desfecho primário estimado pela pesquisa foi a frequência de intercorrências gestacionais e obstétricas no TEA.

O presente estudo foi submetido ao Comitê de Ética em Pesquisa da Plataforma Brasil, possuindo como número de parecer: 1.965.583. O projeto foi aprovado por esse comitê no dia 15 de março de 2017.

Foram respeitadas as normas vigentes para pesquisa em seres humanos segundo a resolução do Ministério da Saúde (MS) e Conselho Nacional de Saúde (CNS), através da Comissão Nacional de Ética em Pesquisa (Conep), Resolução n. 466 de 12 de dezembro de 2012.

\section{RESULTADOS}


Tabela 1 - Percentual e valor bruto das intercorrências gestacionais e obstétricas

Intercorrências gestacionais e obstétricas

Prematuridade

Sangramentos durante gestação

Sofrimento fetal

Tentativas de aborto

Parto pós-termo

Rubéola gestaciona

Doença hipertensiva específica da gravidez

Pré-eclâmpsia

Aspiração meconial

Circulares do cordão umbilical no pescoço

Diabetes gestacional

Oligoidrâmnio

Trabalho de parto $>24 \mathrm{~h}$

Cianose ao nascimento

ITU na gestação

Tabagismo durante gestação

Ameaças de aborto

Queda durante gestação

Restrição de crescimento intrauterino

Episódios de síncope na gestação

Desconforto respiratório ao nascimento

Eclâmpsia

Uso de fórceps no parto

Leiomatose uterina materna durante gestação

Toxoplasmose na gestação

Uso de álcool na gestação

Uso de drogas ilícitas na gestação

Descolamento de placenta

Eritroblastose fetal

Hidrocefalia fetal

Infecção por HPV na gestação

Incompetência istmocervical materna sendo necessário cerclagem do colo

Mielomeningocele fetal

Poli-hidrâmnio

Contrações prematuras

\section{Incidência bruta}

14

12

10

8

8

6

6

5

5

5

5

5

4

4

\section{Porcentagem de incidência}

$16,66 \%$

$14,28 \%$

$11,90 \%$

$9,52 \%$

$9,52 \%$

$8,33 \%$

$7,14 \%$

$7,14 \%$

$5,95 \%$

$5,95 \%$

$5,95 \%$

$5,95 \%$

$5,95 \%$

$4,76 \%$

$4,76 \%$

$4,76 \%$

$3,57 \%$

$3,57 \%$

$3,57 \%$

$3,57 \%$

$2,38 \%$

$2,38 \%$

$2,38 \%$

$2,38 \%$

$2,38 \%$

$2,38 \%$

$2,38 \%$

$1,19 \%$

$1,19 \%$

$1,19 \%$

$1,19 \%$

$1,19 \%$

$1,19 \%$

$1,19 \%$

$1,19 \%$

ITU: infecção do trato urinário; HPV: papilomavírus humano. 
Foram colhidos dados de 209 prontuários médicos, porém foram excluídos 65 por estarem preenchidos de forma incompleta. Desse modo, o número amostral total foi de 144 prontuários.

As idades dos pacientes variaram entre 5 e 30 anos. A faixa etária mais presente foi a de 10 a 15 anos, contendo 57 pacientes, ou seja, 39,6\% de todo o número amostral total. Faixa etária 5-10 anos: $32,6 \% ; 15-20$ anos: $14,6 \%$; 20-25 anos: $11,1 \% ; 25-30$ anos: $2,1 \%$.

Os pacientes do sexo masculino predominaram no estudo, sendo estes em número de 118 contra 26 do sexo feminino.

A etnia predominante na amostra foi a parda, com 69 pacientes, enquanto que a menos comum foi a amarela com apenas um paciente. A etnia negra representou um total de $30,60 \%$ e a etnia branca $20,80 \%$ de todo o número amostral.

Dos 144 pacientes, 60 não tiveram nenhuma intercorrência gestacional ou obstétrica, isto é, em 41,66\% das gestações das crianças autistas não houve intercorrências. As crianças que tiveram algum tipo de intercorrência gestacional ou obstétrica totalizaram 58,33\% .

No grupo com intercorrências gestacionais e obstétricas (84 crianças) ocorreram 35 diferentes alterações, com alguns indivíduos apresentando mais que uma alteração (média de 1,69 alterações por criança). A prematuridade e os sangramentos durante a gestação foram as intercorrências que mais predominaram. Todas as intercorrências e suas incidências em número bruto e em porcentagem relacionadas aos 84 pacientes estão demonstradas na tabela 1.

Em relação ao grau de autismo tivemos um total de 71 pacientes com autismo grave e $73 \mathrm{com}$ autismo leve a moderado, ou seja, $49,30 \%$ de autismo grave contra 50,70\% de autismo leve-moderado entre todos os pacientes. Entre os pacientes com autismo grave, 48 tiveram alguma intercorrência gestacional e obstétrica, correspondendo a $67,60 \%$ contra 23 que não tiveram nenhuma intercorrência, correspondendo a 32,40\% (Figura 1).

Figura 1 - Porcentagem da incidência de intercorrências gestacionais e obstétricas em pacientes com autismo grave

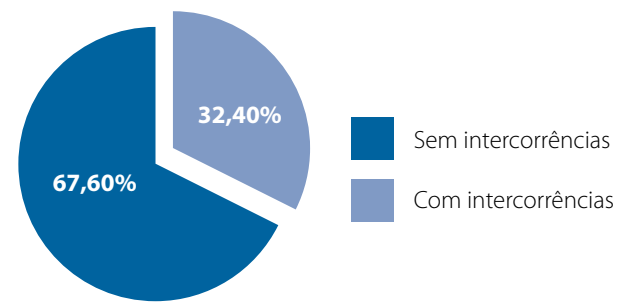

Fonte: Autoria própria (2018)
Entre os pacientes com autismo leve-moderado, 36 deles tiveram alguma intercorrência, correspondendo a $49,3 \%$, contra 37 que não tiveram nenhuma intercorrência gestacional ou obstétrica, correspondendo a $50,7 \%$.

\section{DISCUSSÃO}

Os resultados obtidos serão discutidos nas subseções a seguir, com as intercorrências gestacionais, obstétricas e perinatais mais prevalentes no estudo, sua relação com o grau de autismo, bem como os dados epidemiológicos da população estudada.

\section{DADOS EPIDEMIOLÓGICOS}

Encontramos uma maior prevalência de meninos na população amostral total. Esse dado já era previsível devido a estudos demonstrarem que o autismo é cerca de cinco vezes mais comum no sexo masculino do que no sexo feminino. ${ }^{2}$

Os dados relacionados à etnia demonstraram um maior percentual da etnia parda, seguida de negros, brancos e amarelos, nas crianças autistas. Esses dados são condizentes com o censo epidemiológico da cidade de Salvador realizado pelo Instituto Brasileiro de Geografia e Estatística (IBGE), em 2010, ${ }^{7}$ no qual a maioria dos cidadãos se declararam pardos, seguidos de negros, brancos e, por último, amarelos. Dessa forma, podemos concluir que o TEA ocorre em todas as etnias, mas não podemos inferir acerca de prevalecer mais em uma etnia que em outra. ${ }^{7}$

\section{INTERCORRÊNCIAS GESTACIONAIS, OBSTÉTRICAS E PERINATAIS}

A maioria das crianças autistas pesquisadas tiveram alguma intercorrência em sua gestação e/ou nascimento. Segundo Bryson, Smith e Eastwood (1988), citados por Sanches ${ }^{8}$ em seu estudo utilizando as chamadas 'escalas de ótimo gestacional', a partir de uma escala com intercorrências pré, peri e pós-natal aplicadas em indivíduos autistas e em população-controle, concluiu-se que a média do escore gestacional ótimo total foi menor nos grupos autistas em comparação aos controles. Eles concluíram também que as intercorrências pré-natais são mais frequentes no grupo dos autistas que as relacionadas ao parto e às intercorrências pós-natais. ${ }^{8}$ Dessa forma, os resultados da atual pesquisa estão em concordância com o estudo citado, demonstrando que no período gestacional das crianças autistas parece haver grande número de intercorrências acontecendo.

Outra significativa conclusão é feita por Konstantareas 
(1986), citada por Sanches: ${ }^{8}$ um importante fator relacionado à etiologia do autismo é a ocorrência de danos anatômicos inespecíficos que ocorrem na vida perinatal. Arndt e colaboradores (2005), citados por Sanches, ${ }^{8}$ também sugeriram que a origem do autismo ocorresse ainda no período pré-natal, levando a alterações na anatomia de regiões cerebrais.

Fatores obstétricos e perinatais - como sangramento materno, peso ao nascimento, hipóxia perinatal, prematuridade, dentre outras - têm sido associados ao TEA e são variáveis muito estudadas. Acredita-se que esses fatores contribuam para uma inflamação cerebral focal, o que pode se correlacionar à fisiopatologia do TEA, entretanto ainda sem comprovação.?

A pesquisa de Hallmayer e colaboradores (2011) citados por Langridge et al., ${ }^{5}$ concluiu que os fatores genéticos podem contribuir muito menos do que se pensava na etiologia do autismo, sendo os fatores ambientais responsáveis por pelo menos $50 \%$ das etiologias. ${ }^{5}$

Dessa forma, o resultado do presente estudo no que diz respeito a intercorrências gestacionais e obstétricas terem ocorrido na maioria das crianças autistas pesquisadas faz ligação com os autores referenciados, por se tratar de um fator biológico a que o feto está exposto e que tem potencial de causar danos cerebrais.

Um estudo realizado em Curitiba com 75 crianças com TEA demonstrou alta prevalência de prematuridade, baixo peso ao nascer e hipóxia perinatal nos indivíduos com TEA quando comparados à população geral. ${ }^{9}$

Outros fatores de risco relatados para o desenvolvimento de TEA foram ameaça de aborto, diabetes gestacional, pré-eclâmpsia, parto prematuro e baixo peso ao nascer. ${ }^{5}$

\section{PREMATURIDADE}

A prematuridade foi a intercorrência que mais prevaleceu nos resultados do presente estudo. Nascimento prematuro tem sido descrito como um importante fator de risco para vários problemas de desenvolvimento infantil, entre eles o autismo. ${ }^{10} \mathrm{Um}$ estudo realizado em Atlanta, Geórgia, entre 1981 a 1993, demonstrou que a idade gestacional foi associada ao dobro de aumento de risco para o autismo em nascimentos pré-termo. ${ }^{10}$

A prematuridade está possivelmente associada a um maior risco de TEA. Entretanto, não se sabe se o transtorno está associado à própria prematuridade ou às comorbidades a ela relacionadas, pois recém-nascidos que com o tempo desenvolvem TEA têm taxas altas de complicações perinatais. ${ }^{9}$

\section{SANGRAMENTOS DURANTE A GESTAÇÃO}

A ocorrência de sangramentos durante a gestação foi a segunda intercorrência mais prevalente no grupo estudado. Segundo Gardener et. al, ${ }^{4}$ existe um risco elevado de desenvolver TEA associado ao sangramento materno na gestação, tendo o autor encontrado um risco de $81 \%$ na sua pesquisa.

Um estudo englobando fatores pré-natais no autismo e na população geral concluiu que no grupo com autismo houve maior incidência de sangramento uterino durante a gestação. ${ }^{10}$

Uma falha do presente estudo está relacionada aos sangramentos terem sido relatados de forma retrospectiva pelas mães das crianças autistas, e dessa forma não ser possível classificar o sangramento como uterino ou vaginal.

\section{SOFRIMENTO FETAL}

A presença de sofrimento fetal foi a terceira intercorrência mais relatada nas gestações de crianças com TEA no presente estudo. Aspiração meconial, cianose ao nascimento e desconforto respiratório ao nascimento foram outras intercorrências encontradas e que também estão relacionadas com a diminuição da oxigenação fetal.

Sofrimento fetal, parto prolongado e complicações do cordão são fatores que se acredita estar relacionados a hipóxia fetal e foram relacionados a maior risco de desenvolver autismo. ${ }^{4}$

A associação entre TEA e asfixia perinatal está presente em vários estudos. O sistema dopaminérgico parece ser ativado em excesso após uma anóxia causada pela asfixia neonatal, e em contrapartida algumas crianças autistas possuem hiperatividade dopaminérgica. ${ }^{9}$

\section{INFECÇÕES MATERNAS}

Infecções maternas durante a gravidez, nas formas de infecção do trato urinário, toxoplasmose gestacional e rubéola gestacional, foram descritas nas gestações das crianças autistas pesquisadas no presente estudo.

Existem hipóteses de que certas infecções congênitas estão relacionadas ao desenvolvimento de autismo em crianças que já seriam susceptíveis geneticamente. ${ }^{11}$ Exposição a infecções virais ou bacterianas no útero foram possivelmente relacionadas a falhas no desenvolvimento inicial do cérebro, sendo associadas a um maior risco de autismo. ${ }^{12}$ Infecções, como rubéola e citomegalovírus também foram associadas ao autismo. ${ }^{12}$

Os resultados de uma infecção pré-natal viral, entretanto, dependem de vários fatores, como o estado imunológico materno, a susceptibilidade fetal, o estágio de 
desenvolvimento fetal, a carga viral que atinge o feto, qual o vírus relacionado, dentre outros. ${ }^{12}$

\section{GRAU DE AUTISMO}

Em relação ao grau de autismo, tivemos uma quantidade semelhante de pacientes com autismo grave e autismo leve-moderado. A maioria dos pacientes com autismo grave tiveram alguma intercorrência gestacional e obstétrica.

A heterogeneidade dos fatores ambientais pode levar a subgrupos clínicos com fenótipos cognitivo-comportamentais distintos. ${ }^{13}$ Isso se apoia no experimento feito por Lacaria et al., citado por Tordjman et al., ${ }^{13}$ no qual a partir de um modelo animal concluiu que parece haver um papel nas interações geneambientes na determinação da gravidade do autismo.

\section{LIMITAÇÕES}

Percebemos, então, que diversas intercorrências pré-natais e obstétricas encontradas no presente estudo foram relacionadas de alguma forma com o desenvolvimento do TEA. Se isto significa relação de causa e efeito, o desenho deste estudo não permite concluir. Trata-se de um estudo retrospectivo, com uma amostra de conveniência pequena e sem grupo-controle.

Segundo Tordjman et al., ${ }^{13}$ é importante entender o autismo como um contexto multifatorial, ou seja, como uma organização psicopatológica resultante dos efeitos de vários fatores biológicos, genéticos, ambientais e suas interações.

Nenhum fator de risco no período neonatal e perinatal foi consistentemente validado como desencadeante de autismo. Entretanto, vários fatores foram identificados como associados ao autismo em vários estudos e poderiam ser considerados potencialmente importantes para o desenvolvimento do autismo. ${ }^{14}$

A identificação de fatores ambientais durante a gestação que trazem risco para o desenvolvimento do autismo traz implicações clínicas no que diz respeito à prevenção primária ${ }^{14} \mathrm{e}$ por isso necessita de mais pesquisas.

\section{CONCLUSÕES}

O presente estudo investigou a gestação de 144 crianças autistas acompanhadas em uma escola especializada, em relação a intercorrências gestacionais e obstétricas.

Esta análise permitiu concluir que nas gestações de crianças autistas existe grande concentração de intercorrências acontecendo. Prematuridade, sangramento durante a gestação e sofrimento fetal foram as intercorrências mais prevalentes na gestação das crianças com TEA da escola especializada pesquisada. Crianças com autismo grave tiveram maior percentual de intercorrências gestacionais e obstétricas do que crianças com autismo leve.

\section{REFERÊNCIAS}

1 Associação Americana de Psiquiatria. Manual diagnóstico e estatístico de transtornos mentais - DSM-5. Porto Alegre: Artmed; 2014.

2 Fuentes J, Bakare M, Munir K, Aguayo P, Gaddour N, Öner Ö. Autism Spectrum Disorder. In: Rey JM (Ed). IACAPAP e-textbook of child and adolescent mental health. Geneva: International Association for Child and Adolescent Psychiatry and Allied Professions; 2014. p.1-35.

3 Klin A. Autismo e síndrome de Asperger: uma visão geral. Rev Bras Psiquiatr. 2006;28(Supl I):3-11. DOI: http://dx.doi. org/10.1590/S1516-44462006000500002

4 Gardener H, Spiegelman D, Buka SL. Prenatal risk factors for autism: a comprehensive meta-analysis. Br J Psychiatry. 2009;195(1):7-14. DOI: https://doi.org/10.1192/bjp. bp.108.051672

5 Langridge AT, Glasson EJ, Nassar N, Jacoby P, Pennell C, Hagan Ronald et al. Maternal conditions and perinatal characteristics associated with autism spectrum disorder and intellectual disability. PLoS One. 2013;8(1):e50963. DOI: https://doi. org/10.1371/journal.pone.0050963

6 Pereira AM. Autismo infantil: tradução e validação da CARS (childhood autism rating scale) para uso no Brasil [acesso em 26 nov 2018]. Porto Alegre (RS). Dissertação [Mestrado em Ciências Médicas] - Universidade Federal Rio Grande do Sul; 2007. Disponível em: https://www.lume.ufrgs.br/bitstream/ handle/10183/12936/000634977.pdf

7 Instituto Brasileiro de Geografia e Estatística. Censo

Demográfico de Salvador [acesso em 12 dez 2018]. Salvador: IBGE; 2010. Disponível em: https://cidades.ibge.gov.br/brasil/ ba/salvador/panorama

8 Sanches CP, Brunoni D. Intercorrências perinatais em indivíduos com transtornos invasivos do desenvolvimento: uma revisão [acesso em 12 dez 2018]. Cadernos de PósGraduação em Distúrbios do Desenvolvimento. 2010;10(1):2131. Disponível em: https://www.mackenzie.br/fileadmin/ ARQUIVOS/Public/6-pos-graduacao/upm-higienopolis/ mestrado-doutorado/disturbios_desenvolvimento/2010/ cadernos/1/62118_3.pdf

9 Fezer GF, Matos MB, Nau AL, Zeigelboim BS, Marques JM, Liberalesso PBN. Características perinatais de crianças com transtorno do espectro autista. Rev Paul Pediatr. 2017;35(2):130-5. DOI: http://dx.doi. org/10.1590/1984-0462/;2017;35;2;00003

10 Porto RF, Brunoni D. Transtornos do espectro do autismo: intercorrências perinatais. In: D'Antino ME, Brunoni D, Schwartzman JS (Eds.). Contribuições para a inclusão escolar de alunos com necessidades especiais: estudos interdisciplinares em educação e saúde em alunos com transtorno do espectro do autismo no município de Barueri, SP. São Paulo: Memnon Edições Científicas; 2015. p. 32-41.

11 Fadda MG; Cury VE. O enigma do autismo: contribuições sobre a etiologia do transtorno [acesso em 12 dez 2018]. Psicol Estud. 2016;21(3):411-23. Disponível em: http://periodicos.uem.br/ ojs/index.php/PsicolEstud/article/view/30709

12 Grabrucker AM. Environmental factors in autism. Front 
Psychiatry. 2013;3(118):1-13. DOI: https://doi.org/10.3389/ fpsyt.2012.00118

13 Tordjman S, Somogyi E, Coulon N, Kermarrec S, Cohen D, Bronsard $\mathrm{G}$ et al. Gene $\times$ environment interactions in autism spectrum disorders: role of epigenetic mechanisms. Front Psychiatry. 2014;5(53):1-17. DOI: https://doi.org/10.3389/ fpsyt.2014.00053

14 Guinchat V, Thorsen P, Laurent C, Cans C, Bodeau N, Cohen D. Pre-, peri- and neonatal risk factors for autism. Acta Obstet Gynecol Scand. 2012;91(3):287-300. DOI: https://doi. org/10.1111/j.1600-0412.2011.01325.x 